CRYSTALLOGRAPHIC COMMUNICATIONS

ISSN 2056-9890

Received 16 June 2020

Accepted 19 July 2020

Edited by J. T. Mague, Tulane University, USA

Keywords: crystal structure; Hirshfeld surfaces; quinoxaline; hydrogen bonding.

CCDC reference: 1936664

Supporting information: this article has supporting information at journals.iucr.org/e

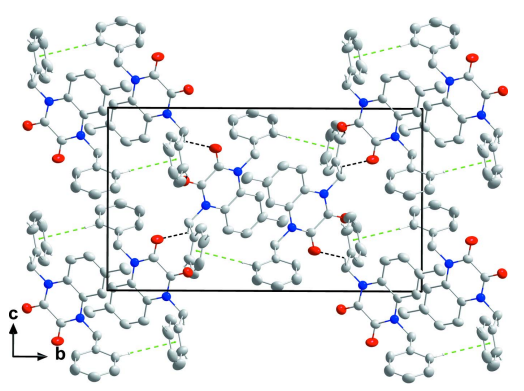

OPEN $\odot$ ACCESS

\section{Synthesis, crystal structures and Hirshfeld surface analysis of 1,4-dibenzyl-6-methyl-1,4-dihydro- quinoxaline-2,3-dione}

\author{
Emine Berrin Cinar, ${ }^{a}$ Ayman Zouitini, ${ }^{\text {b }}$ Youssef Kandri Rodi, ${ }^{\text {b }}$ Younes Ouzidan, \\ Jérôme Marrot, ${ }^{\mathrm{d}}$ Damien Prim, ${ }^{\mathrm{d} *}$ Necmi Dege $^{\mathrm{a}}$ and Eiad Saif ${ }^{\mathrm{e}}$
}

\begin{abstract}
${ }^{\mathbf{a}}$ Department of Physics, Faculty of Arts and Sciences, Ondokuz Mayıs University, Samsun, 55200, Turkey, ${ }^{\mathbf{b}}$ Laboratoire de Chimie Organique Appliquée, Université Sidi Mohamed Ben Abdallah, Faculté des Sciences et Techniques, BP 2202, Fez, Morocco, 'Laboratoire de Chimie Physique et Chimie Bio-organique, Faculté des Sciences et Techniques Mohammedia, Université Hassan II, Casablanca, BP 146, 28800, Mohammedia, Morocco, Institut Lavoisier de Versailles, UVSQ, CNRS, Université Paris-Saclay, 78035 Versailles, France, and ${ }^{\mathbf{e}}$ Department of Computer and Electronic Engineering Technology, Sana'a Community College, Sana'a, Yemen. *Correspondence e-mail:

eiad.saif2016@gmail.com
\end{abstract}

The title quinoxaline molecule, $\mathrm{C}_{23} \mathrm{H}_{20} \mathrm{~N}_{2} \mathrm{O}_{2}$, is not planar, the dihedral angle angle between the mean planes of the benzene rings being $72.54(15)^{\circ}$. In the crystal, molecules are connected into chains extending parallel to (101) by weak $\mathrm{C}-\mathrm{H} \cdots \mathrm{O}$ hydrogen bonds. Weak $\mathrm{C}-\mathrm{H} \cdots \pi$ interactions link the chains, forming a three-dimensional network structure. Hirshfeld surface analysis revealed that the most important contributions for the crystal packing are from $\mathrm{H} \cdots \mathrm{H}(48.7 \%), \mathrm{H} \cdots \mathrm{C} / \mathrm{C} \cdots \mathrm{H}(32.0 \%), \mathrm{H} \cdots \mathrm{O} / \mathrm{O} \cdots \mathrm{H}(15.4 \%), \mathrm{C} \cdots \mathrm{C}(1.9 \%)$, $\mathrm{H} \cdots \mathrm{N} / \mathrm{N} \cdots \mathrm{H}(1.1 \%)$ contacts.

\section{Chemical context}

Given their importance in the pharmaceutical, chemical and industrial fields, the synthesis of quinoxaline and its derivatives has been a goal of chemists in recent years. Quinoxaline derivatives find use as anticancer (Noolvi et al., 2011), antimalarial (Guillon et al., 2004), antifungal (Xu \& Fan, 2011), antiviral (Cai et al., 2008) and anti-inflammatory (Yan et al., 2007) agents. Some quinoxaline derivatives have also been reported to be corrosion inhibitors for steel in an acidic medium (Zouitini et al., 2018, 2019; El Janati et al., 2020). In this work, we report the synthesis and structure of the title compound obtained by the action of benzyl chloride on 6-methyl-1,4-dihydroquinoxaline-2,3-dione in the presence of potassium carbonate and a catalytic quantity of tetra- $n$ butylammonium bromide. A Hirshfeld surface analysis was also performed.

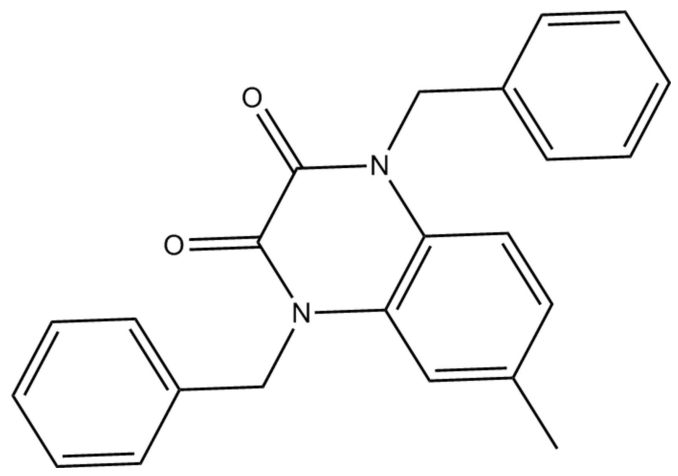


Table 1

Hydrogen-bond geometry $\left(\AA,^{\circ}\right)$.

$\mathrm{Cg} 3$ is the centroid of the $\mathrm{C} 11-\mathrm{C} 16$ benzene ring.

\begin{tabular}{lllll}
\hline$D-\mathrm{H} \cdots A$ & $D-\mathrm{H}$ & $\mathrm{H} \cdots A$ & $D \cdots A$ & $D-\mathrm{H} \cdots A$ \\
\hline $\mathrm{C} 10-\mathrm{H} 10 A \cdots \mathrm{O} 2^{\mathrm{i}}$ & 0.97 & 2.52 & $3.251(3)$ & 132 \\
$\mathrm{C} 16-\mathrm{H} 16 \cdots \mathrm{O} 1^{\mathrm{i}}$ & 0.93 & 2.55 & $3.3984(4)$ & 152 \\
$\mathrm{C} 25-\mathrm{H} 25 \cdots C g 3^{\mathrm{ii}}$ & 0.93 & 2.83 & $3.683(3)$ & 153 \\
\hline
\end{tabular}

Symmetry codes: (i) $x-\frac{1}{2},-y+\frac{1}{2}, z-\frac{1}{2}$; (ii) $-x+2,-y,-z$.

\section{Structural commentary}

An ORTEPIII (Burnett \& Johnson, 1996) view of the molecule is given in Fig. 1. The molecule is not planar, the dihedral angle angle between the mean planes of the benzene rings (C11-C16 and C24-C29) being $72.54(15)^{\circ}$. The mean planes of the $\mathrm{C} 1 / \mathrm{N} 2 / \mathrm{C} 9 / \mathrm{C} 8 / \mathrm{N} 1 / \mathrm{C} 6$ and $\mathrm{C} 11-\mathrm{C} 16$ rings make an angle of $73.093(13)^{\circ}$ while the $\mathrm{C} 1-\mathrm{C} 6$ and $\mathrm{C} 24-\mathrm{C} 29$ rings make an angle of $79.01(14)^{\circ}$. The $\mathrm{C} 1 / \mathrm{N} 2 / \mathrm{C} 9 / \mathrm{C} 8 / \mathrm{N} 1 / \mathrm{C} 6$ and $\mathrm{C} 1-\mathrm{C} 6$ rings are nearly coplanar, subtending a dihedral angle of only $3.07(11)^{\circ}$. The $\mathrm{C} 8=\mathrm{O} 1$ and $\mathrm{C} 9=\mathrm{O} 2$ bonds show double-bond character with bond lengths of $1.222(3)$ and 1.217 (3) $\AA$, respectively. The $\mathrm{N} 1-\mathrm{C} 10$ and $\mathrm{N} 2-\mathrm{C} 23$ bond lengths are 1.476 (3) and 1.464 (3) $\AA$, respectively while the $\mathrm{C} 11-\mathrm{C} 10-$ $\mathrm{N} 1$ bond angle is $113.57(18)^{\circ}$ and the $\mathrm{N} 2-\mathrm{C} 23-\mathrm{C} 24$ bond angle is $114.05(18)^{\circ}$. The $\mathrm{C} 9-\mathrm{N} 2-\mathrm{C} 23-\mathrm{C} 24$ and $\mathrm{C} 8-\mathrm{N} 1-$ $\mathrm{C} 10-\mathrm{C} 11$ torsion angles are $-96.7(2)$ and $-93.4(2)^{\circ}$, respectively.

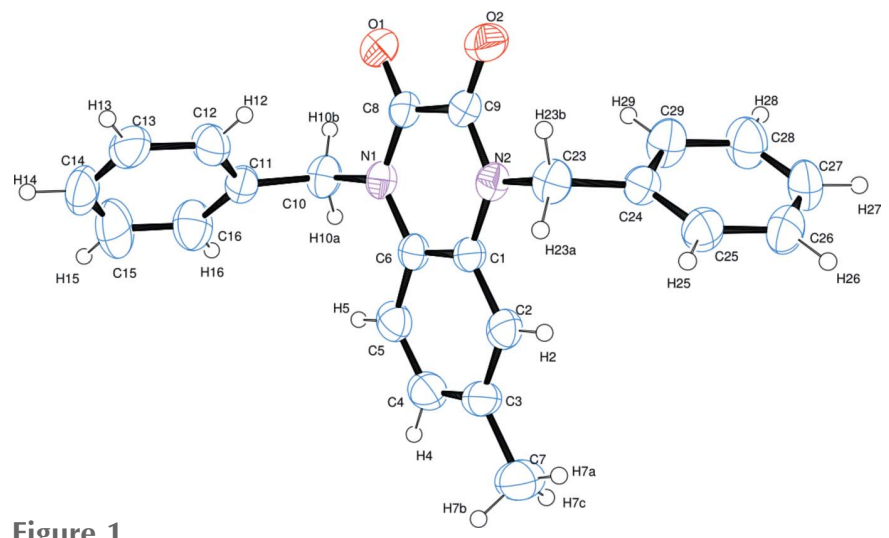

The molecular structure of the title compound. Displacement ellipsoids are drawn at the $40 \%$ probability level.

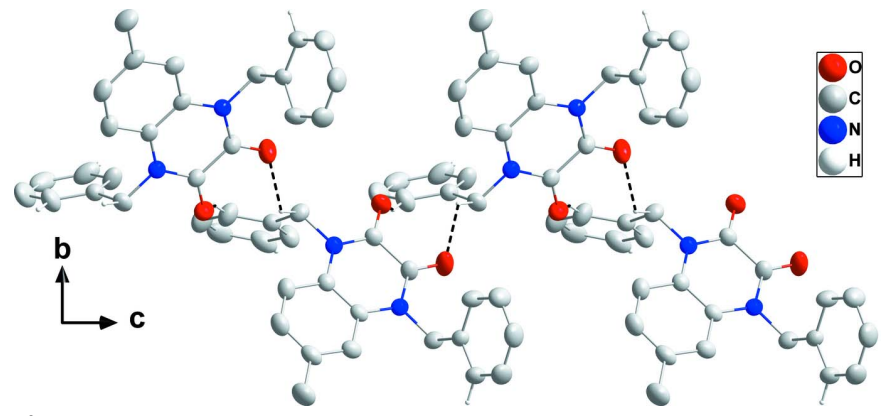

Figure 2

View of a portion of a chain along the $a$-axis direction with $\mathrm{C}-\mathrm{H} \cdots \mathrm{O}$ hydrogen bonds depicted by dashed lines.

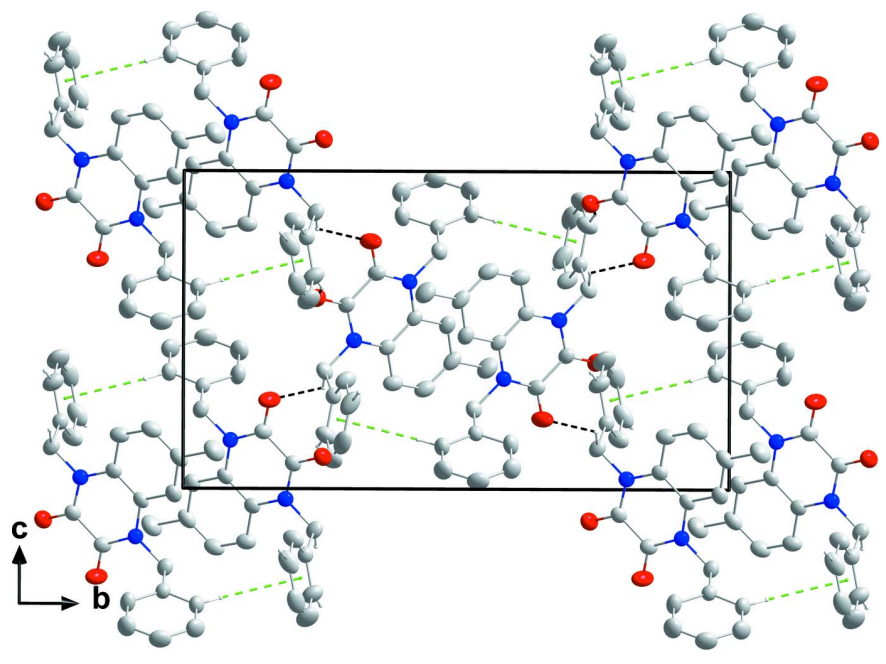

Figure 3

Packing viewed along the $a$-axis direction with $\mathrm{C}-\mathrm{H} \cdots \mathrm{O}$ hydrogen bonds and $\mathrm{C}-\mathrm{H} \cdots \pi$ (ring) interactions depicted, respectively, by black and green dashed lines.

\section{Supramolecular features}

In the crystal, molecules are connected by weak $\mathrm{C} 16-$ $\mathrm{H} 16 \cdots \mathrm{O} 1$ and $\mathrm{C} 10-\mathrm{H} 10 A \cdots \mathrm{O} 2$ hydrogen bonds into chains extending parallel to (101) (Table 1 and Fig. 2). Weak C25$\mathrm{H} 25 \cdots C g 3$ interactions $(2.83 \AA$; $C g 3$ is the centroid of the C11-C16 ring at $-x+2,-y,-z$ ) link the chains into a threedimensional network structure (Table 1 and Fig. 3).

\section{Hirshfeld surface analysis}

The CrystalExplorer17.5 (Turner et al., 2017) program was used to analyse the interactions within the crystal. The donoracceptor groups are visualized using a standard (high) surface resolution and $d_{\text {norm }}$ surfaces mapped over a fixed colour scale of -0.140 (red) to 1.358 (blue) a.u., as illustrated in Fig. 4. Red spots on the surface of the $d_{\text {norm }}$ plot indicate intermolecular contacts involving the hydrogen bonds. The red spots identified in Fig. 4(a) correspond to the intermolecular $\mathrm{C}-\mathrm{H} \cdots \mathrm{O}$ bonds. Regions close to the sum of the van der Waals radii are shown in white. Fig. 4(b) shows the shape-index surface, which can be used to detect the presence of $\pi$-stacking interactions.
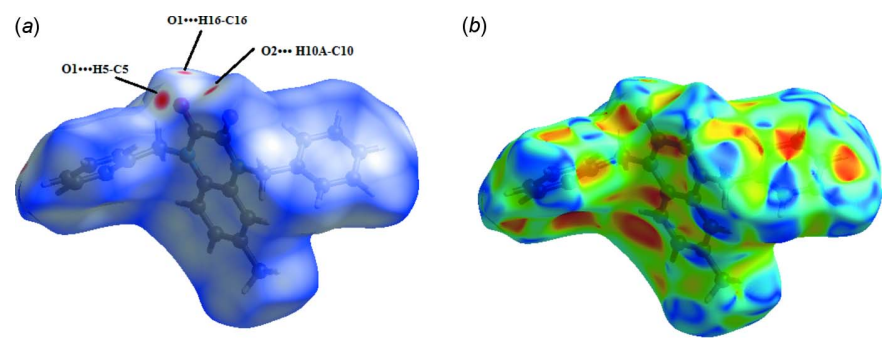

Figure 4

Hirshfeld surface mapper over $(a) d_{\text {norm }}$ and $(b)$ shape-index to visualize the interactions in the title compound. 

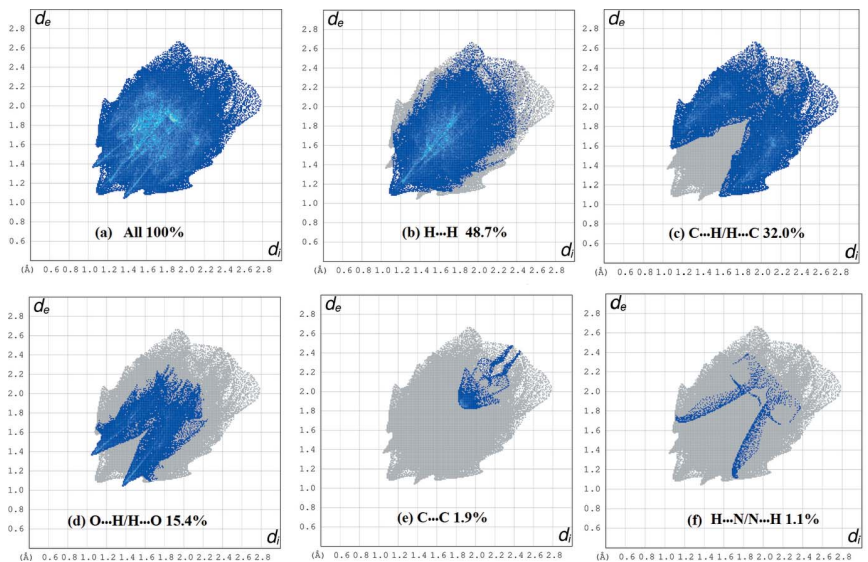

Figure 5

Fingerprint plot for all interactions and those delineated into the most important interactions.

The absence of characteristic triangles indicates that no significant $\pi-\pi$ interactions are present. Two-dimensional fingerprints were also generated in the range -1 to $1 \AA$ (Fig. 5). As expected, $\mathrm{H} \cdot \mathrm{H}(48.7 \%)$ and $\mathrm{H} \cdot \mathrm{C} / \mathrm{C} \cdot \mathrm{H}$ $(32.0 \%)$ contacts dominate the intermolecular interactions, but the $\mathrm{O} \cdots \mathrm{H} / \mathrm{H} \cdots \mathrm{O}$ contacts are important directional intermolecular interactions in the crystal. The C..C $(1.9 \%)$ and $\mathrm{H} \cdot \cdots \mathrm{N} / \mathrm{N} \cdots \mathrm{H}(1.1 \%)$ contribute minimally to the overall crystal packing.

\section{Database survey}

A search of the Cambridge Structural Database (CSD, version 5.40, update August 2019; Groom et al., 2016) using 1-benzyl3,4 -dihydroquinoxalin-2(1H)-one as the main skeleton revealed the presence of three structures similar to the title compound, but with different substituents. These are: 1,4,6tribenzoyl-3-(4-bromobenzyl)-1,4-dihydroquinoxaline-2-one (LEQWIO; Abraham et al., 2006), 1,4-dibenzoyl-6-trifluoromethyl-3-(4-bromobenzyl)-1,4-dihydroquinoxaline-2-one (LEQWOU; Abraham et al., 2006) and 1,4-dibenzyl-6-chloro1,4-dihydroquinoxaline-2,3-dione (PAWFEB; El Janati et al., 2017). In the latter study (PAWFEB) examining compounds having the same skeletal system as the 1,4-dihydroquinoxaline-2,3-dione structure in the title compound, the corrosion inhibition efficiency of 1,4-diallyl-6-chloroquinoxaline-2,3-(1H,4H)-dione and 1,4-diallyl-6-nitroquinoxaline2,3-(1H,4H)-dione on mild steel (MS) in $1.0 \mathrm{M} \mathrm{HCl}$ solution was investigated.

\section{Synthesis and crystallization}

To a solution of 6-methyl-1,4-dihydroquinoxaline-2,3-dione $(0.3 \mathrm{~g}, 1.73 \mathrm{mmol})$ in DMF $(15 \mathrm{~mL})$, were added potassium carbonate $(0.47 \mathrm{~g}, 3.61 \mathrm{mmol})$ and tetra- $n$ butylammonium bromide $(0.07 \mathrm{~g}, 0.23 \mathrm{mmol})$. After stirring for $10 \mathrm{~min}, 0.5 \mathrm{~mL}$ (4.32 mmol) of benzyl chloride was added and the mixture was stirred at room temperature for $6 \mathrm{~h}$. After filtration of the salts, the DMF was evaporated under reduced pressure and
Table 2

Experimental details.

\begin{tabular}{|c|c|}
\hline \multicolumn{2}{|l|}{ Crystal data } \\
\hline Chemical formula & $\mathrm{C}_{23} \mathrm{H}_{20} \mathrm{~N}_{2} \mathrm{O}_{2}$ \\
\hline$M_{\mathrm{r}}$ & 356.41 \\
\hline Crystal system, space group & Monoclinic, $P 2_{1} / n$ \\
\hline Temperature $(\mathrm{K})$ & 296 \\
\hline$a, b, c(\AA)$ & $\begin{array}{l}9.0844(12), 18.7227(18), \\
\quad 11.2708(14)\end{array}$ \\
\hline$\beta\left({ }^{\circ}\right)$ & $104.848(4)$ \\
\hline$V\left(\mathrm{\AA}^{3}\right)$ & $1853.0(4)$ \\
\hline$Z$ & 4 \\
\hline Radiation type & Мо $K \alpha$ \\
\hline$\mu\left(\mathrm{mm}^{-1}\right)$ & 0.08 \\
\hline Crystal size $(\mathrm{mm})$ & $0.30 \times 0.16 \times 0.06$ \\
\hline \multicolumn{2}{|l|}{ Data collection } \\
\hline Diffractometer & Bruker APEXII CCD \\
\hline Absorption correction & $\begin{array}{l}\text { Multi-scan (SADABS; Bruker, } \\
\text { 2016) }\end{array}$ \\
\hline$T_{\min }, T_{\max }$ & $0.677,0.746$ \\
\hline $\begin{array}{l}\text { No. of measured, independent and } \\
\text { observed }[I>2 \sigma(I)] \text { reflections }\end{array}$ & $22287,4253,2487$ \\
\hline$R_{\text {int }}$ & 0.054 \\
\hline$(\sin \theta / \lambda)_{\max }\left(\AA^{-1}\right)$ & 0.649 \\
\hline \multicolumn{2}{|l|}{ Refinement } \\
\hline$R\left[F^{2}>2 \sigma\left(F^{2}\right)\right], w R\left(F^{2}\right), S$ & $0.060,0.188,1.04$ \\
\hline No. of reflections & 4253 \\
\hline No. of parameters & 244 \\
\hline No. of restraints & 1 \\
\hline H-atom treatment & $\mathrm{H}$-atom parameters constrained \\
\hline$\Delta \rho_{\max }, \Delta \rho_{\min }\left(\mathrm{e} \AA^{-3}\right)$ & $0.38,-0.25$ \\
\hline
\end{tabular}

Computer programs: APEX3 and SAINT (Bruker, 2016), SHELXT2018/3 (Sheldrick, 2015a), SHELXL2018/3 (Sheldrick, 2015b), OLEX2 (Dolomanov et al., 2009), Mercury (Macrae et al., 2020), WinGX (Farrugia, 2012), PLATON (Spek, 2020) and publCIF (Westrip, 2010).

the residue obtained was dissolved in dichloromethane. The organic phase was then dried over $\mathrm{Na}_{2} \mathrm{SO}_{4}$ and concentrated. The mixture obtained was chromatographed on a silica gel column [eluent: hexane/ethylacetate (2/1)]. The crude product was recrystallized from ethanol as yellow crystals suitable for X-ray analysis (m.p. $493.5 \mathrm{~K}$ ).

\section{Refinement}

Crystal data, data collection and structure refinement details are summarized in Table 2. Hydrogen atoms treated as riding: $\mathrm{C}-\mathrm{H}=0.97 \AA$ and $U_{\text {iso }}(\mathrm{H})=1.5 U_{\text {eq }}(\mathrm{C})$ for methyl, $\mathrm{C}-\mathrm{H}=$ $0.96 \AA$ and $U_{\text {iso }}(\mathrm{H})=1.2 U_{\text {eq }}(\mathrm{C})$ for methylene, $\mathrm{C}-\mathrm{H}=0.93 \AA$ and $U_{\text {iso }}(\mathrm{H})=1.2 U_{\text {eq }}(\mathrm{C})$ for aromatic and $\mathrm{C}-\mathrm{H}=0.98 \AA$ and $U_{\text {iso }}(\mathrm{H})=1.2 U_{\text {eq }}(\mathrm{C})$ for methine $\mathrm{H}$ atoms.

\section{Funding information}

This study was supported by Ondokuz Mayıs University under project No. PYOFEN.1906.19.001.

\section{References}

Abraham, C. J., Paull, D. H., Scerba, M. T., Grebinski, J. W. \& Lectka, T. (2006). J. Am. Chem. Soc. 128, 13370-13371.

Bruker (2016). APEX3, SADABS and SAINT. Bruker AXS Inc., Madison, Wisconsin, USA.

Burnett, M. N. \& Johnson, C. K. (1996). ORTEPIII. Report ORNL6895. Oak Ridge National Laboratory, Tennessee, USA. 
Cai, J., Zou, J., Pan, X. \& Zhang, W. (2008). Tetrahedron Lett. 49, 7386-7390.

Dolomanov, O. V., Bourhis, L. J., Gildea, R. J., Howard, J. A. K. \& Puschmann, H. (2009). J. Appl. Cryst. 42, 339-341.

El Janati, A., Elmsellem, H., Kandri Rodi, Y., Ouzidan, Y., Ramdani, M., Mokhtari, M., Abdel-Rahman, I., Cherif Alaoui, I., Ouazzani Chahdi, F. \& Kusuma, H. S. (2020). Int. J. Corros. Scale Inhib. 9, 644-660.

El Janati, A., Kandri Rodi, Y., Jasinski, J. P., Kaur, M., Ouzidan, Y. \& Essassi, E. M. (2017). IUCrData, 2, x170901.

Farrugia, L. J. (2012). J. Appl. Cryst. 45, 849-854.

Groom, C. R., Bruno, I. J., Lightfoot, M. P. \& Ward, S. C. (2016). Acta Cryst. B72, 171-179.

Guillon, J., Grellier, P., Labaied, M., Sonnet, P., Léger, J. M., DéprezPoulain, R., Forfar-Bares, I., Dallemagne, P., Lemaitre, N., Péhourcq, F., Rochette, J., Sergheraert, C. \& Jarry, C. (2004). J. Med. Chem. 47, 1997-2009.

Macrae, C. F., Sovago, I., Cottrell, S. J., Galek, P. T. A., McCabe, P., Pidcock, E., Platings, M., Shields, G. P., Stevens, J. S., Towler, M. \& Wood, P. A. (2020). J. Appl. Cryst. 53, 226-235.
Noolvi, M. N., Patel, H. M., Bhardwaj, V. \& Chauhan, A. (2011). Eur. J. Med. Chem. 46, 2327-2346.

Sheldrick, G. M. (2015a). Acta Cryst. A71, 3-8.

Sheldrick, G. M. (2015b). Acta Cryst. C71, 3-8.

Spek, A. L. (2020). Acta Cryst. E76, 1-11.

Turner, M. J., Mckinnon, J. J., Wolff, S. K., Grimwood, D. J., Spackman, P. R., Jayatilaka, D. \& Spackman, M. A. (2017). CrystalExplorer17.5. The University of Western Australia.

Westrip, S. P. (2010). J. Appl. Cryst. 43, 920-925.

Xu, H. \& Fan, L. L. (2011). Eur. J. Med. Chem. 46, 1919-1925.

Yan, L., Liu, F. W., Dai, G. F. \& Liu, H. M. (2007). Bioorg. Med. Chem. Lett. 17, 609-612.

Zouitini, A., Kandri Rodi, Y., Elmselem, H., Ouazzani Chahdi, F., Steli, H., Ad, C., Ouzidan, Y., Essassi, E., Chetouani, A. \& Hammouti, B. (2018). Moroc. J. Chem. 6, 391-403.

Zouitini, A., Kandri Rodi, Y., Ouzidan, Y., Ouazzani Chahdi, F., Mokhtari, M., Abdel-Rahman, I., Essassi, E. M., Aouniti, A., Hammouti, B. \& Elmsellem, H. (2019). Int. J. Corros. Scale Inhib. 8, $225-240$. 


\section{supporting information}

Acta Cryst. (2020). E76, 1361-1364 [https://doi.org/10.1107/S2056989020009895]

Synthesis, crystal structures and Hirshfeld surface analysis of 1,4-dibenzyl-6methyl-1,4-dihydroquinoxaline-2,3-dione

\section{Emine Berrin Cinar, Ayman Zouitini, Youssef Kandri Rodi, Younes Ouzidan, Jérôme Marrot, Damien Prim, Necmi Dege and Eiad Saif}

Computing details

Data collection: APEX3 (Bruker, 2016); cell refinement: SAINT (Bruker, 2016); data reduction: SAINT (Bruker, 2016); program(s) used to solve structure: SHELXT2018/3 (Sheldrick, 2015a); program(s) used to refine structure:

SHELXL2018/3 (Sheldrick, 2015b); molecular graphics: OLEX2 (Dolomanov et al., 2009) and Mercury (Macrae et al., 2020); software used to prepare material for publication: WinGX (Farrugia, 2012), PLATON (Spek, 2020), SHELXL2018 (Sheldrick, 2015b) and publCIF (Westrip, 2010).

1,4-Dibenzyl-6-methyl-1,4-dihydroquinoxaline-2,3-dione

Crystal data

$\mathrm{C}_{23} \mathrm{H}_{20} \mathrm{~N}_{2} \mathrm{O}_{2}$

$M_{r}=356.41$

Monoclinic, $P 2_{1} / n$

$a=9.0844(12) \AA$

$b=18.7227(18) \AA$

$c=11.2708(14) \AA$

$\beta=104.848(4)^{\circ}$

$V=1853.0(4) \AA^{3}$

$Z=4$

Data collection

Bruker APEXII CCD

diffractometer

Radiation source: fine-focus sealed tube

$\varphi$ and $\omega$ scans

Absorption correction: multi-scan

(SADABS; Bruker, 2016)

$T_{\min }=0.677, T_{\max }=0.746$

22287 measured reflections

\section{Refinement}

Refinement on $F^{2}$

Least-squares matrix: full

$R\left[F^{2}>2 \sigma\left(F^{2}\right)\right]=0.060$

$w R\left(F^{2}\right)=0.188$

$S=1.04$

4253 reflections

244 parameters
$F(000)=752$

$D_{\mathrm{x}}=1.278 \mathrm{Mg} \mathrm{m}^{-3}$

Mo $K \alpha$ radiation, $\lambda=0.71073 \AA$

Cell parameters from 4963 reflections

$\theta=2.6-24.6^{\circ}$

$\mu=0.08 \mathrm{~mm}^{-1}$

$T=296 \mathrm{~K}$

Parallelepiped, yellow

$0.30 \times 0.16 \times 0.06 \mathrm{~mm}$

4253 independent reflections

2487 reflections with $I>2 \sigma(I)$

$R_{\text {int }}=0.054$

$\theta_{\max }=27.5^{\circ}, \theta_{\min }=2.2^{\circ}$

$h=-10 \rightarrow 11$

$k=-17 \rightarrow 24$

$l=-14 \rightarrow 14$

1 restraint

Primary atom site location: structure-invariant direct methods

Secondary atom site location: difference Fourier map

Hydrogen site location: inferred from neighbouring sites 
H-atom parameters constrained

$w=1 /\left[\sigma^{2}\left(F_{\mathrm{o}}^{2}\right)+(0.0761 P)^{2}+0.7497 P\right]$

where $P=\left(F_{\mathrm{o}}^{2}+2 F_{\mathrm{c}}^{2}\right) / 3$

$$
\begin{aligned}
& (\Delta / \sigma)_{\max }<0.001 \\
& \Delta \rho_{\max }=0.38 \mathrm{e} \AA^{-3} \\
& \Delta \rho_{\min }=-0.24 \mathrm{e} \AA^{-3}
\end{aligned}
$$

Special details

Geometry. All esds (except the esd in the dihedral angle between two 1.s. planes) are estimated using the full covariance matrix. The cell esds are taken into account individually in the estimation of esds in distances, angles and torsion angles; correlations between esds in cell parameters are only used when they are defined by crystal symmetry. An approximate

\begin{tabular}{|c|c|c|c|c|}
\hline & $x$ & $y$ & $z$ & $U_{\text {iso }} * / U_{\text {eq }}$ \\
\hline O1 & $0.6364(2)$ & $0.24430(9)$ & $0.60030(16)$ & $0.0668(5)$ \\
\hline $\mathrm{O} 2$ & $0.6604(2)$ & $0.34222(11)$ & $0.77924(17)$ & $0.0787(6)$ \\
\hline $\mathrm{C} 1$ & $0.3801(2)$ & $0.42320(11)$ & $0.5380(2)$ & $0.0453(5)$ \\
\hline $\mathrm{C} 2$ & $0.2840(3)$ & $0.48294(13)$ & $0.5167(2)$ & $0.0565(6)$ \\
\hline $\mathrm{H} 2$ & 0.290850 & 0.516663 & 0.578499 & $0.068^{*}$ \\
\hline $\mathrm{C} 3$ & 0.1779 & $0.49239(14)$ & $0.4035(3)$ & $0.0606(7)$ \\
\hline $\mathrm{C} 4$ & 0.1685 & $0.44202(16)$ & $0.3149(3)$ & $0.0665(7)$ \\
\hline H4 & 0.096780 & 0.447513 & 0.240048 & $0.080 *$ \\
\hline $\mathrm{C} 5$ & 0.2611 & $0.38383(14)$ & $0.3329(2)$ & $0.0605(7)$ \\
\hline H5 & 0.253041 & 0.350819 & 0.269920 & $0.073 *$ \\
\hline C6 & $0.3690(2)$ & $0.37294(12)$ & $0.4457(2)$ & $0.0463(5)$ \\
\hline $\mathrm{C} 7$ & $0.0744(4)$ & $0.55686(17)$ & $0.3816(3)$ & $0.0857(9)$ \\
\hline $\mathrm{H} 7 \mathrm{~A}$ & 0.097017 & 0.586438 & 0.453541 & $0.129 *$ \\
\hline H7B & 0.090205 & 0.583627 & 0.313222 & $0.129 *$ \\
\hline $\mathrm{H} 7 \mathrm{C}$ & -0.029766 & 0.541485 & 0.364004 & $0.129 *$ \\
\hline N1 & $0.4634(2)$ & $0.31227(10)$ & $0.46724(17)$ & $0.0479(5)$ \\
\hline $\mathrm{C} 8$ & 0.5589 & $0.29843(12)$ & $0.5791(2)$ & $0.0502(6)$ \\
\hline $\mathrm{C} 9$ & 0.5729 & $0.35335(13)$ & $0.6795(2)$ & $0.0530(6)$ \\
\hline $\mathrm{N} 2$ & $0.4870(2)$ & $0.41352(10)$ & $0.65252(17)$ & $0.0477(5)$ \\
\hline $\mathrm{C} 10$ & $0.4593(3)$ & $0.25871(12)$ & $0.3703(2)$ & $0.0564(6)$ \\
\hline $\mathrm{H} 10 \mathrm{~A}$ & 0.356426 & 0.255943 & 0.318086 & $0.068^{*}$ \\
\hline $\mathrm{H} 10 \mathrm{~B}$ & 0.484757 & 0.212320 & 0.408395 & $0.068 *$ \\
\hline C11 & $0.5669(3)$ & $0.27494(12)$ & $0.2918(2)$ & $0.0520(6)$ \\
\hline $\mathrm{C} 12$ & $0.7141(3)$ & $0.29739(15)$ & $0.3413(3)$ & $0.0682(7)$ \\
\hline H12 & 0.746539 & 0.306858 & 0.424992 & $0.082 *$ \\
\hline $\mathrm{C} 13$ & $0.8143(4)$ & $0.30607(17)$ & $0.2690(3)$ & $0.0839(10)$ \\
\hline H13 & 0.913384 & 0.321355 & 0.303781 & $0.101 *$ \\
\hline $\mathrm{C} 14$ & $0.7672(5)$ & $0.29203(17)$ & $0.1452(4)$ & 0.0907 (11) \\
\hline H14 & 0.834725 & 0.297218 & 0.096117 & $0.109 *$ \\
\hline $\mathrm{C} 15$ & $0.6232(6)$ & $0.2708(2)$ & $0.0954(3)$ & 0.1045 (12) \\
\hline H15 & 0.590820 & 0.262012 & 0.011500 & $0.125^{*}$ \\
\hline C16 & $0.5229(4)$ & $0.26191(17)$ & $0.1682(3)$ & $0.0820(9)$ \\
\hline H16 & 0.423893 & 0.246823 & 0.132573 & $0.098 *$ \\
\hline $\mathrm{C} 23$ & $0.5078(3)$ & $0.46790(13)$ & $0.7488(2)$ & $0.0561(6)$ \\
\hline $\mathrm{H} 23 \mathrm{~A}$ & 0.502294 & 0.514721 & 0.711069 & $0.067^{*}$ \\
\hline H23B & 0.608828 & 0.462678 & 0.803360 & $0.067 *$ \\
\hline
\end{tabular}
(isotropic) treatment of cell esds is used for estimating esds involving 1.s. planes.

Fractional atomic coordinates and isotropic or equivalent isotropic displacement parameters $\left(\hat{A}^{2}\right)$ 


$\begin{array}{lllll}\mathrm{C} 24 & 0.3920(3) & 0.46424(11) & 0.8239(2) & 0.0486(5) \\ \mathrm{C} 25 & 0.3412(3) & 0.52590(13) & 0.8661(2) & 0.0641(7) \\ \mathrm{H} 25 & 0.373901 & 0.569841 & 0.844097 & 0.077^{*} \\ \mathrm{C} 26 & 0.2429(4) & 0.52371(16) & 0.9403(3) & 0.0785(9) \\ \mathrm{H} 26 & 0.208237 & 0.565956 & 0.967137 & 0.094^{*} \\ \mathrm{C} 27 & 0.1958(4) & 0.45942(18) & 0.9747(3) & 0.0799(9) \\ \mathrm{H} 27 & 0.129675 & 0.457886 & 1.025374 & 0.096^{*} \\ \mathrm{C} 28 & 0.2455(4) & 0.39791(16) & 0.9349(3) & 0.0777(8) \\ \mathrm{H} 28 & 0.214200 & 0.354182 & 0.959034 & 0.093^{*} \\ \mathrm{C} 29 & 0.3424(3) & 0.39995(13) & 0.8588(3) & 0.0666(7) \\ \mathrm{H} 29 & 0.374561 & 0.357497 & 0.830698 & 0.080^{*}\end{array}$

Atomic displacement parameters $\left(\AA^{2}\right)$

\begin{tabular}{|c|c|c|c|c|c|c|}
\hline & $U^{11}$ & $U^{22}$ & $U^{33}$ & $U^{12}$ & $U^{13}$ & $U^{23}$ \\
\hline $\mathrm{O} 1$ & $0.0702(12)$ & $0.0659(11)$ & $0.0686(12)$ & $0.0218(9)$ & $0.0256(9)$ & $0.0050(8)$ \\
\hline $\mathrm{O} 2$ & $0.0710(13)$ & $0.1036(15)$ & $0.0554(11)$ & $0.0349(11)$ & $0.0050(10)$ & $-0.0041(10)$ \\
\hline $\mathrm{C} 1$ & $0.0359(11)$ & $0.0508(12)$ & $0.0539(14)$ & $0.0000(9)$ & $0.0197(10)$ & $0.0130(10)$ \\
\hline $\mathrm{C} 2$ & $0.0536(14)$ & $0.0563(13)$ & $0.0671(16)$ & $0.0013(11)$ & $0.0292(13)$ & $0.0090(12)$ \\
\hline $\mathrm{C} 3$ & $0.0433(13)$ & $0.0678(15)$ & $0.0736(18)$ & $0.0060(11)$ & $0.0204(13)$ & $0.0259(14)$ \\
\hline $\mathrm{C} 4$ & $0.0497(15)$ & $0.089(2)$ & $0.0604(16)$ & 0.0034 (14) & $0.0131(12)$ & $0.0162(15)$ \\
\hline $\mathrm{C} 5$ & $0.0476(14)$ & $0.0820(17)$ & $0.0528(15)$ & $-0.0043(13)$ & $0.0149(12)$ & $0.0033(13)$ \\
\hline C6 & $0.0370(11)$ & $0.0550(12)$ & $0.0514(13)$ & $-0.0041(10)$ & $0.0197(10)$ & $0.0057(10)$ \\
\hline C7 & $0.071(2)$ & $0.090(2)$ & $0.094(2)$ & $0.0186(17)$ & $0.0178(17)$ & $0.0233(18)$ \\
\hline N1 & $0.0414(10)$ & $0.0542(11)$ & $0.0525(12)$ & $-0.0031(8)$ & $0.0200(9)$ & $0.0006(9)$ \\
\hline $\mathrm{C} 8$ & $0.0458(13)$ & $0.0563(13)$ & $0.0540(14)$ & $0.0056(11)$ & $0.0231(11)$ & 0.0057 (11) \\
\hline C9 & $0.0449(13)$ & $0.0656(15)$ & $0.0518(14)$ & $0.0104(11)$ & $0.0184(12)$ & $0.0029(11)$ \\
\hline N2 & $0.0442(10)$ & $0.0510(10)$ & $0.0519(11)$ & $0.0002(8)$ & $0.0194(9)$ & $-0.0012(8)$ \\
\hline $\mathrm{C} 10$ & $0.0487(14)$ & $0.0588(14)$ & $0.0650(16)$ & $-0.0127(11)$ & $0.0204(12)$ & $-0.0112(12)$ \\
\hline C11 & $0.0514(14)$ & $0.0517(12)$ & $0.0570(14)$ & $0.0012(11)$ & $0.0213(11)$ & $-0.0011(11)$ \\
\hline $\mathrm{C} 12$ & $0.0526(15)$ & $0.0883(19)$ & $0.0666(17)$ & $-0.0084(14)$ & $0.0205(13)$ & $0.0175(14)$ \\
\hline $\mathrm{C} 13$ & $0.0586(17)$ & 0.087 (2) & $0.117(3)$ & $0.0116(15)$ & $0.0420(18)$ & $0.0379(19)$ \\
\hline $\mathrm{C} 14$ & $0.116(3)$ & $0.0689(18)$ & $0.117(3)$ & $0.0319(19)$ & $0.084(3)$ & $0.0182(18)$ \\
\hline $\mathrm{C} 15$ & $0.143(4)$ & 0.109 (3) & $0.082(2)$ & $-0.003(3)$ & $0.066(3)$ & $-0.024(2)$ \\
\hline $\mathrm{C} 16$ & $0.087(2)$ & $0.094(2)$ & $0.0682(19)$ & $-0.0083(17)$ & $0.0272(16)$ & $-0.0236(16)$ \\
\hline $\mathrm{C} 23$ & $0.0534(14)$ & $0.0531(13)$ & $0.0644(15)$ & $-0.0076(11)$ & $0.0198(12)$ & $-0.0054(11)$ \\
\hline $\mathrm{C} 24$ & $0.0478(13)$ & $0.0474(12)$ & $0.0513(13)$ & $-0.0012(10)$ & $0.0142(10)$ & $-0.0025(10)$ \\
\hline $\mathrm{C} 25$ & $0.0786(19)$ & $0.0498(13)$ & $0.0684(17)$ & 0.0069 (12) & $0.0272(15)$ & $-0.0007(12)$ \\
\hline $\mathrm{C} 26$ & $0.099(2)$ & $0.0731(18)$ & $0.0750(19)$ & $0.0230(17)$ & $0.0426(17)$ & $-0.0042(15)$ \\
\hline $\mathrm{C} 27$ & $0.082(2)$ & $0.106(2)$ & $0.0638(18)$ & $0.0093(18)$ & $0.0411(16)$ & $0.0004(17)$ \\
\hline $\mathrm{C} 28$ & $0.090(2)$ & $0.0710(18)$ & $0.085(2)$ & $-0.0113(16)$ & $0.0457(17)$ & $0.0041(15)$ \\
\hline $\mathrm{C} 29$ & $0.0773(18)$ & $0.0492(13)$ & $0.0837(19)$ & $-0.0066(12)$ & $0.0396(15)$ & $-0.0059(13)$ \\
\hline
\end{tabular}

Geometric parameters $\left(\AA,{ }^{\circ}\right)$

\begin{tabular}{llll}
\hline $\mathrm{O} 1-\mathrm{C} 8$ & $1.222(3)$ & $\mathrm{C} 16-\mathrm{H} 16$ & 0.9300 \\
$\mathrm{O} 2-\mathrm{C} 9$ & $1.217(3)$ & $\mathrm{C} 15-\mathrm{C} 14$ & $1.345(5)$ \\
$\mathrm{C} 1-\mathrm{C} 6$ & $1.388(3)$ & $\mathrm{C} 15-\mathrm{H} 15$ & 0.9300
\end{tabular}




\begin{tabular}{|c|c|c|c|}
\hline $\mathrm{C} 1-\mathrm{C} 2$ & $1.401(3)$ & $\mathrm{C} 14-\mathrm{C} 13$ & $1.376(5)$ \\
\hline $\mathrm{C} 1-\mathrm{N} 2$ & $1.414(3)$ & $\mathrm{C} 14-\mathrm{H} 14$ & 0.9300 \\
\hline $\mathrm{C} 2-\mathrm{C} 3$ & $1.400(4)$ & $\mathrm{C} 13-\mathrm{C} 12$ & $1.378(4)$ \\
\hline $\mathrm{C} 2-\mathrm{H} 2$ & 0.9300 & C13-H13 & 0.9300 \\
\hline $\mathrm{C} 3-\mathrm{C} 4$ & $1.360(4)$ & $\mathrm{C} 12-\mathrm{H} 12$ & 0.9300 \\
\hline $\mathrm{C} 3-\mathrm{C} 7$ & $1.511(4)$ & $\mathrm{C} 23-\mathrm{C} 24$ & $1.511(3)$ \\
\hline $\mathrm{C} 4-\mathrm{C} 5$ & $1.360(4)$ & $\mathrm{C} 23-\mathrm{H} 23 \mathrm{~A}$ & 0.9700 \\
\hline $\mathrm{C} 4-\mathrm{H} 4$ & 0.9300 & $\mathrm{C} 23-\mathrm{H} 23 \mathrm{~B}$ & 0.9700 \\
\hline $\mathrm{C} 5-\mathrm{C} 6$ & $1.407(3)$ & $\mathrm{C} 24-\mathrm{C} 25$ & $1.373(3)$ \\
\hline $\mathrm{C} 5-\mathrm{H} 5$ & 0.9300 & $\mathrm{C} 24-\mathrm{C} 29$ & $1.378(3)$ \\
\hline $\mathrm{C} 6-\mathrm{N} 1$ & $1.406(3)$ & $\mathrm{C} 29-\mathrm{C} 28$ & $1.378(4)$ \\
\hline $\mathrm{N} 1-\mathrm{C} 8$ & $1.359(3)$ & $\mathrm{C} 29-\mathrm{H} 29$ & 0.9300 \\
\hline $\mathrm{N} 1-\mathrm{C} 10$ & $1.476(3)$ & $\mathrm{C} 28-\mathrm{C} 27$ & $1.355(4)$ \\
\hline $\mathrm{C} 8-\mathrm{C} 9$ & $1.510(3)$ & $\mathrm{C} 28-\mathrm{H} 28$ & 0.9300 \\
\hline $\mathrm{C} 9-\mathrm{N} 2$ & $1.360(3)$ & $\mathrm{C} 27-\mathrm{C} 26$ & $1.366(4)$ \\
\hline $\mathrm{N} 2-\mathrm{C} 23$ & $1.464(3)$ & $\mathrm{C} 27-\mathrm{H} 27$ & 0.9300 \\
\hline $\mathrm{C} 10-\mathrm{C} 11$ & $1.507(3)$ & $\mathrm{C} 26-\mathrm{C} 25$ & $1.371(4)$ \\
\hline $\mathrm{C} 10-\mathrm{H} 10 \mathrm{~A}$ & 0.9700 & $\mathrm{C} 26-\mathrm{H} 26$ & 0.9300 \\
\hline $\mathrm{C} 10-\mathrm{H} 10 \mathrm{~B}$ & 0.9700 & $\mathrm{C} 25-\mathrm{H} 25$ & 0.9300 \\
\hline $\mathrm{C} 11-\mathrm{C} 16$ & $1.370(4)$ & C7-H7A & 0.9600 \\
\hline $\mathrm{C} 11-\mathrm{C} 12$ & $1.376(4)$ & C7-H7B & 0.9600 \\
\hline $\mathrm{C} 16-\mathrm{C} 15$ & $1.384(5)$ & $\mathrm{C} 7-\mathrm{H} 7 \mathrm{C}$ & 0.9600 \\
\hline $\mathrm{C} 6-\mathrm{C} 1-\mathrm{C} 2$ & $119.4(2)$ & $\mathrm{C} 14-\mathrm{C} 15-\mathrm{C} 16$ & $120.4(3)$ \\
\hline $\mathrm{C} 6-\mathrm{C} 1-\mathrm{N} 2$ & $119.92(19)$ & $\mathrm{C} 14-\mathrm{C} 15-\mathrm{H} 15$ & 119.8 \\
\hline $\mathrm{C} 2-\mathrm{C} 1-\mathrm{N} 2$ & $120.7(2)$ & $\mathrm{C} 16-\mathrm{C} 15-\mathrm{H} 15$ & 119.8 \\
\hline $\mathrm{C} 3-\mathrm{C} 2-\mathrm{C} 1$ & $120.7(2)$ & $\mathrm{C} 15-\mathrm{C} 14-\mathrm{C} 13$ & $119.7(3)$ \\
\hline $\mathrm{C} 3-\mathrm{C} 2-\mathrm{H} 2$ & 119.7 & $\mathrm{C} 15-\mathrm{C} 14-\mathrm{H} 14$ & 120.1 \\
\hline $\mathrm{C} 1-\mathrm{C} 2-\mathrm{H} 2$ & 119.7 & $\mathrm{C} 13-\mathrm{C} 14-\mathrm{H} 14$ & 120.1 \\
\hline $\mathrm{C} 4-\mathrm{C} 3-\mathrm{C} 2$ & $118.7(2)$ & $\mathrm{C} 14-\mathrm{C} 13-\mathrm{C} 12$ & $119.8(3)$ \\
\hline $\mathrm{C} 4-\mathrm{C} 3-\mathrm{C} 7$ & $121.0(3)$ & $\mathrm{C} 14-\mathrm{C} 13-\mathrm{H} 13$ & 120.1 \\
\hline $\mathrm{C} 2-\mathrm{C} 3-\mathrm{C} 7$ & $120.3(3)$ & $\mathrm{C} 12-\mathrm{C} 13-\mathrm{H} 13$ & 120.1 \\
\hline $\mathrm{C} 5-\mathrm{C} 4-\mathrm{C} 3$ & $121.7(3)$ & $\mathrm{C} 11-\mathrm{C} 12-\mathrm{C} 13$ & $121.1(3)$ \\
\hline $\mathrm{C} 5-\mathrm{C} 4-\mathrm{H} 4$ & 119.1 & $\mathrm{C} 11-\mathrm{C} 12-\mathrm{H} 12$ & 119.4 \\
\hline $\mathrm{C} 3-\mathrm{C} 4-\mathrm{H} 4$ & 119.1 & $\mathrm{C} 13-\mathrm{C} 12-\mathrm{H} 12$ & 119.4 \\
\hline $\mathrm{C} 4-\mathrm{C} 5-\mathrm{C} 6$ & $120.8(3)$ & $\mathrm{N} 2-\mathrm{C} 23-\mathrm{C} 24$ & 114.05 (18) \\
\hline $\mathrm{C} 4-\mathrm{C} 5-\mathrm{H} 5$ & 119.6 & $\mathrm{~N} 2-\mathrm{C} 23-\mathrm{H} 23 \mathrm{~A}$ & 108.7 \\
\hline $\mathrm{C} 6-\mathrm{C} 5-\mathrm{H} 5$ & 119.6 & $\mathrm{C} 24-\mathrm{C} 23-\mathrm{H} 23 \mathrm{~A}$ & 108.7 \\
\hline $\mathrm{C} 1-\mathrm{C} 6-\mathrm{N} 1$ & $119.6(2)$ & $\mathrm{N} 2-\mathrm{C} 23-\mathrm{H} 23 \mathrm{~B}$ & 108.7 \\
\hline $\mathrm{C} 1-\mathrm{C} 6-\mathrm{C} 5$ & $118.6(2)$ & $\mathrm{C} 24-\mathrm{C} 23-\mathrm{H} 23 \mathrm{~B}$ & 108.7 \\
\hline $\mathrm{N} 1-\mathrm{C} 6-\mathrm{C} 5$ & $121.7(2)$ & $\mathrm{H} 23 \mathrm{~A}-\mathrm{C} 23-\mathrm{H} 23 \mathrm{~B}$ & 107.6 \\
\hline $\mathrm{C} 8-\mathrm{N} 1-\mathrm{C} 6$ & $122.01(19)$ & $\mathrm{C} 25-\mathrm{C} 24-\mathrm{C} 29$ & $118.2(2)$ \\
\hline $\mathrm{C} 8-\mathrm{N} 1-\mathrm{C} 10$ & $116.47(19)$ & $\mathrm{C} 25-\mathrm{C} 24-\mathrm{C} 23$ & $120.0(2)$ \\
\hline $\mathrm{C} 6-\mathrm{N} 1-\mathrm{C} 10$ & $121.47(19)$ & $\mathrm{C} 29-\mathrm{C} 24-\mathrm{C} 23$ & $121.7(2)$ \\
\hline $\mathrm{O} 1-\mathrm{C} 8-\mathrm{N} 1$ & $122.6(2)$ & $\mathrm{C} 28-\mathrm{C} 29-\mathrm{C} 24$ & $120.7(2)$ \\
\hline $\mathrm{O} 1-\mathrm{C} 8-\mathrm{C} 9$ & $118.9(2)$ & $\mathrm{C} 28-\mathrm{C} 29-\mathrm{H} 29$ & 119.7 \\
\hline $\mathrm{N} 1-\mathrm{C} 8-\mathrm{C} 9$ & $118.5(2)$ & $\mathrm{C} 24-\mathrm{C} 29-\mathrm{H} 29$ & 119.7 \\
\hline $\mathrm{O} 2-\mathrm{C} 9-\mathrm{N} 2$ & $123.4(2)$ & $\mathrm{C} 27-\mathrm{C} 28-\mathrm{C} 29$ & $120.2(3)$ \\
\hline
\end{tabular}




\begin{tabular}{|c|c|c|c|}
\hline $\mathrm{O} 2-\mathrm{C} 9-\mathrm{C} 8$ & $119.1(2)$ & $\mathrm{C} 27-\mathrm{C} 28-\mathrm{H} 28$ & 119.9 \\
\hline $\mathrm{N} 2-\mathrm{C} 9-\mathrm{C} 8$ & $117.6(2)$ & $\mathrm{C} 29-\mathrm{C} 28-\mathrm{H} 28$ & 119.9 \\
\hline $\mathrm{C} 9-\mathrm{N} 2-\mathrm{C} 1$ & $122.08(19)$ & $\mathrm{C} 28-\mathrm{C} 27-\mathrm{C} 26$ & $120.0(3)$ \\
\hline $\mathrm{C} 9-\mathrm{N} 2-\mathrm{C} 23$ & $116.9(2)$ & $\mathrm{C} 28-\mathrm{C} 27-\mathrm{H} 27$ & 120.0 \\
\hline $\mathrm{C} 1-\mathrm{N} 2-\mathrm{C} 23$ & $121.01(19)$ & $\mathrm{C} 26-\mathrm{C} 27-\mathrm{H} 27$ & 120.0 \\
\hline $\mathrm{N} 1-\mathrm{C} 10-\mathrm{C} 11$ & $113.57(18)$ & $\mathrm{C} 27-\mathrm{C} 26-\mathrm{C} 25$ & $119.9(2)$ \\
\hline $\mathrm{N} 1-\mathrm{C} 10-\mathrm{H} 10 \mathrm{~A}$ & 108.9 & $\mathrm{C} 27-\mathrm{C} 26-\mathrm{H} 26$ & 120.0 \\
\hline $\mathrm{C} 11-\mathrm{C} 10-\mathrm{H} 10 \mathrm{~A}$ & 108.9 & $\mathrm{C} 25-\mathrm{C} 26-\mathrm{H} 26$ & 120.0 \\
\hline $\mathrm{N} 1-\mathrm{C} 10-\mathrm{H} 10 \mathrm{~B}$ & 108.9 & $\mathrm{C} 26-\mathrm{C} 25-\mathrm{C} 24$ & $121.0(2)$ \\
\hline $\mathrm{C} 11-\mathrm{C} 10-\mathrm{H} 10 \mathrm{~B}$ & 108.9 & $\mathrm{C} 26-\mathrm{C} 25-\mathrm{H} 25$ & 119.5 \\
\hline $\mathrm{H} 10 \mathrm{~A}-\mathrm{C} 10-\mathrm{H} 10 \mathrm{~B}$ & 107.7 & $\mathrm{C} 24-\mathrm{C} 25-\mathrm{H} 25$ & 119.5 \\
\hline $\mathrm{C} 16-\mathrm{C} 11-\mathrm{C} 12$ & $117.8(2)$ & $\mathrm{C} 3-\mathrm{C} 7-\mathrm{H} 7 \mathrm{~A}$ & 109.5 \\
\hline $\mathrm{C} 16-\mathrm{C} 11-\mathrm{C} 10$ & $119.8(2)$ & $\mathrm{C} 3-\mathrm{C} 7-\mathrm{H} 7 \mathrm{~B}$ & 109.5 \\
\hline $\mathrm{C} 12-\mathrm{C} 11-\mathrm{C} 10$ & $122.2(2)$ & $\mathrm{H} 7 \mathrm{~A}-\mathrm{C} 7-\mathrm{H} 7 \mathrm{~B}$ & 109.5 \\
\hline $\mathrm{C} 11-\mathrm{C} 16-\mathrm{C} 15$ & $121.2(3)$ & $\mathrm{C} 3-\mathrm{C} 7-\mathrm{H} 7 \mathrm{C}$ & 109.5 \\
\hline $\mathrm{C} 11-\mathrm{C} 16-\mathrm{H} 16$ & 119.4 & $\mathrm{H} 7 \mathrm{~A}-\mathrm{C} 7-\mathrm{H} 7 \mathrm{C}$ & 109.5 \\
\hline $\mathrm{C} 15-\mathrm{C} 16-\mathrm{H} 16$ & 119.4 & $\mathrm{H} 7 \mathrm{~B}-\mathrm{C} 7-\mathrm{H} 7 \mathrm{C}$ & 109.5 \\
\hline $\mathrm{C} 6-\mathrm{C} 1-\mathrm{C} 2-\mathrm{C} 3$ & $-0.2(3)$ & $\mathrm{C} 6-\mathrm{C} 1-\mathrm{N} 2-\mathrm{C} 9$ & $-5.1(3)$ \\
\hline $\mathrm{N} 2-\mathrm{C} 1-\mathrm{C} 2-\mathrm{C} 3$ & $-179.82(19)$ & $\mathrm{C} 2-\mathrm{C} 1-\mathrm{N} 2-\mathrm{C} 9$ & $174.47(19)$ \\
\hline $\mathrm{C} 1-\mathrm{C} 2-\mathrm{C} 3-\mathrm{C} 4$ & $0.8(3)$ & $\mathrm{C} 6-\mathrm{C} 1-\mathrm{N} 2-\mathrm{C} 23$ & $176.09(18)$ \\
\hline $\mathrm{C} 1-\mathrm{C} 2-\mathrm{C} 3-\mathrm{C} 7$ & $179.8(2)$ & $\mathrm{C} 2-\mathrm{C} 1-\mathrm{N} 2-\mathrm{C} 23$ & $-4.3(3)$ \\
\hline $\mathrm{C} 2-\mathrm{C} 3-\mathrm{C} 4-\mathrm{C} 5$ & $-1.3(4)$ & $\mathrm{C} 8-\mathrm{N} 1-\mathrm{C} 10-\mathrm{C} 11$ & $-93.4(2)$ \\
\hline $\mathrm{C} 7-\mathrm{C} 3-\mathrm{C} 4-\mathrm{C} 5$ & $179.7(2)$ & $\mathrm{C} 6-\mathrm{N} 1-\mathrm{C} 10-\mathrm{C} 11$ & $88.9(2)$ \\
\hline $\mathrm{C} 3-\mathrm{C} 4-\mathrm{C} 5-\mathrm{C} 6$ & $1.2(4)$ & $\mathrm{N} 1-\mathrm{C} 10-\mathrm{C} 11-\mathrm{C} 16$ & $-141.3(2)$ \\
\hline $\mathrm{C} 2-\mathrm{C} 1-\mathrm{C} 6-\mathrm{N} 1$ & $-178.95(18)$ & $\mathrm{N} 1-\mathrm{C} 10-\mathrm{C} 11-\mathrm{C} 12$ & $44.0(3)$ \\
\hline $\mathrm{N} 2-\mathrm{C} 1-\mathrm{C} 6-\mathrm{N} 1$ & $0.6(3)$ & $\mathrm{C} 12-\mathrm{C} 11-\mathrm{C} 16-\mathrm{C} 15$ & $0.2(4)$ \\
\hline $\mathrm{C} 2-\mathrm{C} 1-\mathrm{C} 6-\mathrm{C} 5$ & $0.1(3)$ & $\mathrm{C} 10-\mathrm{C} 11-\mathrm{C} 16-\mathrm{C} 15$ & $-174.7(3)$ \\
\hline $\mathrm{N} 2-\mathrm{C} 1-\mathrm{C} 6-\mathrm{C} 5$ & $179.71(18)$ & $\mathrm{C} 11-\mathrm{C} 16-\mathrm{C} 15-\mathrm{C} 14$ & $0.5(5)$ \\
\hline $\mathrm{C} 4-\mathrm{C} 5-\mathrm{C} 6-\mathrm{C} 1$ & $-0.6(3)$ & $\mathrm{C} 16-\mathrm{C} 15-\mathrm{C} 14-\mathrm{C} 13$ & $-1.0(5)$ \\
\hline $\mathrm{C} 4-\mathrm{C} 5-\mathrm{C} 6-\mathrm{N} 1$ & $178.5(2)$ & $\mathrm{C} 15-\mathrm{C} 14-\mathrm{C} 13-\mathrm{C} 12$ & $0.8(5)$ \\
\hline $\mathrm{C} 1-\mathrm{C} 6-\mathrm{N} 1-\mathrm{C} 8$ & $4.6(3)$ & $\mathrm{C} 16-\mathrm{C} 11-\mathrm{C} 12-\mathrm{C} 13$ & $-0.4(4)$ \\
\hline $\mathrm{C} 5-\mathrm{C} 6-\mathrm{N} 1-\mathrm{C} 8$ & $-174.4(2)$ & $\mathrm{C} 10-\mathrm{C} 11-\mathrm{C} 12-\mathrm{C} 13$ & $174.4(2)$ \\
\hline $\mathrm{C} 1-\mathrm{C} 6-\mathrm{N} 1-\mathrm{C} 10$ & $-177.85(18)$ & $\mathrm{C} 14-\mathrm{C} 13-\mathrm{C} 12-\mathrm{C} 11$ & $-0.1(4)$ \\
\hline $\mathrm{C} 5-\mathrm{C} 6-\mathrm{N} 1-\mathrm{C} 10$ & $3.1(3)$ & $\mathrm{C} 9-\mathrm{N} 2-\mathrm{C} 23-\mathrm{C} 24$ & $-96.7(2)$ \\
\hline $\mathrm{C} 6-\mathrm{N} 1-\mathrm{C} 8-\mathrm{O} 1$ & $176.7(2)$ & $\mathrm{C} 1-\mathrm{N} 2-\mathrm{C} 23-\mathrm{C} 24$ & $82.2(2)$ \\
\hline $\mathrm{C} 10-\mathrm{N} 1-\mathrm{C} 8-\mathrm{O} 1$ & $-1.0(3)$ & $\mathrm{N} 2-\mathrm{C} 23-\mathrm{C} 24-\mathrm{C} 25$ & $-144.8(2)$ \\
\hline $\mathrm{C} 6-\mathrm{N} 1-\mathrm{C} 8-\mathrm{C} 9$ & $-5.3(3)$ & $\mathrm{N} 2-\mathrm{C} 23-\mathrm{C} 24-\mathrm{C} 29$ & $39.5(3)$ \\
\hline $\mathrm{C} 10-\mathrm{N} 1-\mathrm{C} 8-\mathrm{C} 9$ & $177.02(19)$ & $\mathrm{C} 25-\mathrm{C} 24-\mathrm{C} 29-\mathrm{C} 28$ & $-0.6(4)$ \\
\hline $\mathrm{O} 1-\mathrm{C} 8-\mathrm{C} 9-\mathrm{O} 2$ & $-0.4(3)$ & $\mathrm{C} 23-\mathrm{C} 24-\mathrm{C} 29-\mathrm{C} 28$ & $175.1(3)$ \\
\hline $\mathrm{N} 1-\mathrm{C} 8-\mathrm{C} 9-\mathrm{O} 2$ & $-178.5(2)$ & $\mathrm{C} 24-\mathrm{C} 29-\mathrm{C} 28-\mathrm{C} 27$ & $1.2(5)$ \\
\hline $\mathrm{O} 1-\mathrm{C} 8-\mathrm{C} 9-\mathrm{N} 2$ & $179.0(2)$ & $\mathrm{C} 29-\mathrm{C} 28-\mathrm{C} 27-\mathrm{C} 26$ & $-0.6(5)$ \\
\hline $\mathrm{N} 1-\mathrm{C} 8-\mathrm{C} 9-\mathrm{N} 2$ & $0.9(3)$ & $\mathrm{C} 28-\mathrm{C} 27-\mathrm{C} 26-\mathrm{C} 25$ & $-0.4(5)$ \\
\hline $\mathrm{O} 2-\mathrm{C} 9-\mathrm{N} 2-\mathrm{C} 1$ & $-176.4(2)$ & $\mathrm{C} 27-\mathrm{C} 26-\mathrm{C} 25-\mathrm{C} 24$ & $1.0(5)$ \\
\hline $\mathrm{C} 8-\mathrm{C} 9-\mathrm{N} 2-\mathrm{C} 1$ & $4.2(3)$ & $\mathrm{C} 29-\mathrm{C} 24-\mathrm{C} 25-\mathrm{C} 26$ & $-0.4(4)$ \\
\hline $\mathrm{O} 2-\mathrm{C} 9-\mathrm{N} 2-\mathrm{C} 23$ & $2.4(3)$ & $\mathrm{C} 23-\mathrm{C} 24-\mathrm{C} 25-\mathrm{C} 26$ & $-176.2(3)$ \\
\hline $\mathrm{C} 8-\mathrm{C} 9-\mathrm{N} 2-\mathrm{C} 23$ & $-176.91(19)$ & & \\
\hline
\end{tabular}


Hydrogen-bond geometry $\left(A,{ }^{\circ}\right)$

$\mathrm{Cg} 3$ is the centroid of the $\mathrm{C} 11-\mathrm{C} 16$ benzene ring.

\begin{tabular}{lllll}
\hline$D-\mathrm{H} \cdots A$ & $D-\mathrm{H}$ & $\mathrm{H} \cdots A$ & $D \cdots A$ & $D-\mathrm{H} \cdots A$ \\
\hline $\mathrm{C} 10-\mathrm{H} 10 A \cdots \mathrm{O} 2^{\mathrm{i}}$ & 0.97 & 2.52 & $3.251(3)$ & 132 \\
$\mathrm{C} 16-\mathrm{H} 16 \cdots \mathrm{O}^{\mathrm{i}}$ & 0.93 & 2.55 & $3.3984(4)$ & 152 \\
$\mathrm{C} 25-\mathrm{H} 25 \cdots C g 3^{\mathrm{ii}}$ & 0.93 & 2.83 & $3.683(3)$ & 153 \\
\hline
\end{tabular}

Symmetry codes: (i) $x-1 / 2,-y+1 / 2, z-1 / 2$; (ii) $-x+2,-y,-z$. 